\title{
Hierarchical effects of choice-related activity and neural encoding during feature selective attention
}

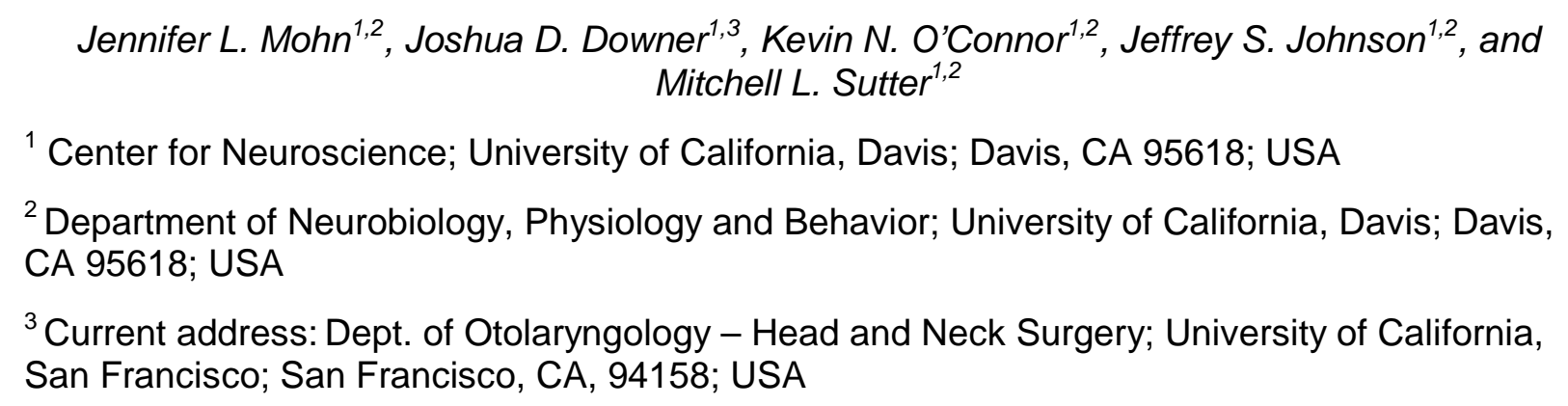

${ }^{1}$ Center for Neuroscience; University of California, Davis; Davis, CA 95618; USA

${ }^{2}$ Department of Neurobiology, Physiology and Behavior; University of California, Davis; Davis, CA 95618; USA

${ }^{3}$ Current address: Dept. of Otolaryngology - Head and Neck Surgery; University of California, San Francisco; San Francisco, CA, 94158; USA

11

\section{Corresponding Author:}

Mitchell L. Sutter

Center for Neuroscience

University of California, Davis

1544 Newton Court Davis CA 95618

mlsutter@ucdavis.edu

No. of pages: 35

No. of Figures (Main): 6

No. of Tables: 1

No. of words: Abstract (222); Introduction (962); Results (1127) Discussion (1479)

Conflict of Interest: The authors declare no competing financial interest

Acknowledgements: This work was funded by NIH NIDCD grant DC002514 (MLS), NSF

GRFP 1148897 (JDD) and ARCS Foundation Fellowship (JDD) 


\section{Abstract}

Selective attention is necessary to sift through, form a coherent percept of, and make behavioral decisions on the vast amount of information present in most sensory environments. How and where selective attention is employed in cortex and how this perceptual information then informs the relevant behavioral decisions is still not well understood. Studies probing selective attention and decision making in visual cortex have been enlightening as to how sensory attention might work in that modality; whether or not similar mechanisms are employed in auditory attention is not yet clear. Therefore, we trained rhesus macaques on a feature selective attention task, where they switched between reporting changes in temporal (amplitude modulation, AM) and spectral (carrier bandwidth) features of a broadband noise stimulus. We investigated how the encoding of these features by single neurons in primary (A1) and secondary (lateral belt, ML) auditory cortex were affected by the different attention conditions. We found that neurons in A1 and ML showed mixedselectivity to the sound and task features. We found no difference in AM encoding between the attention conditions. We found that choice-related activity in both $\mathrm{A} 1$ and $\mathrm{ML}$ neurons shifts between attentional conditions. This finding suggests that choice-related activity in auditory cortex does not simply reflect motor preparation or action, and supports the relationship between reported choice-related activity and the decision and perceptual process. 
bioRxiv preprint doi: https://doi.org/10.1101/2020.03 10.986349; this version posted June 29, 2020. The copyright holder for this preprint (which was not certified by peer review) is the author/funder, who has granted bioRxiv a license to display the preprint in perpetuity. It is made available under aCC-BY-NC-ND 4.0 International license.

\section{New \& Noteworthy}

53 We recorded from primary and secondary auditory cortex while monkeys performed a non-spatial

54 feature attention task. Both areas exhibited rate-based choice-related activity. The manifestation of

55 choice-related activity was attention-dependent, suggesting that choice-related activity in auditory

56 cortex does not simply reflect arousal or motor influences, but relates to the specific perceptual choice.

57 The lack of temporal-based choice activity is consistent with growing evidence that subcortical, but not

58 cortical, single neurons inform decisions through temporal envelope following. 
Introduction

The auditory system is often faced with the difficult challenge of encoding a specific sound in a noisy environment, such as following a conversation in a loud room. The neural mechanisms by which the auditory system attends to one sound source and ignores distracting sounds are not yet understood.

63 Studies probing the mechanisms underlying auditory attention in cortex have been largely concerned

64 with task engagement, wherein the effects of active performance on neural activity is compared to those of passive listening. Studies in auditory cortex $(\mathrm{AC})$ utilizing this paradigm have shown that task engagement can improve behaviorally-relevant neural sound discrimination (Atiani et al. 2014; Bagur et al. 2018; Buran et al. 2014; Carcea et al. 2017; Francis et al. 2018a; Niwa et al. 2012a, 2015; von Trapp et al. 2016), modulate neuronal tuning (Fritz et al. 2003, 2007; Fritz 2005; Lee and Middlebrooks 2011; Lin et al. 2019; Yin et al. 2014), alter the structure of correlated variability within neural populations

70 (Downer et al. 2015, 2017a), and more (Massoudi et al. 2014; Angeloni and Geffen 2018; Osmanski and

71 Wang 2015; Sutter and Shamma 2011). Though informative, this active/passive paradigm makes it

72 difficult to disentangle arousal and motor effects from the mechanisms more specifically employed in

73 selectively attending to a single sound source or feature amidst auditory 'clutter'. unexplored (Downer et al. 2017b). Feature-selective attention, which segregates particular sound features, such as intensity or fundamental frequency, is essential for tasks such as discriminating between talkers in a noisy environment (Bregman 1994; McDermott 2009; Bizley and Cohen 2013; 
In visual cortex, feature-based attention has been suggested to follow a gain model similar to

83 spatial attention, where responsivity to the attended feature increases in cells tuned to the attended

84 feature and decreases in cells tuned to orthogonal features (Martinez-Trujillo and Treue 2004; Maunsell

85 and Treue 2006). Studies of spatial attention in AC single neurons suggest that AC employs a mechanism

86 similar to that reported in visual cortex, where a gain in neural activity increases when attention is

87 directed into the receptive field of a neuron and, conversely, gain decreases when attention is directed outside the receptive field (Engle and Recanzone 2013; Lee and Middlebrooks 2011; Scott et al. 2007) .

We endeavored to see if feature-selective attention in AC is also facilitated by a gain in activity in neurons tuned to an attended feature.

How and where task relevant sensory information is transformed into a decision in the brain is

92 still largely unclear. There have been mixed reports of activity correlated to the reported decision in AC

93 (Christison-Lagay et al. 2017; Elgueda et al. 2019; Guo et al. 2019; Niwa et al. 2012b; Runyan et al. 2017;

94 Tsunada et al. 2016; Tsunada and Cohen 2014). This choice-related activity has been reported in some

95 studies as early as primary auditory cortex (A1) (Atiani et al. 2014; Bathellier et al. 2012; Bizley et al.

96 2013; Christison-Lagay et al. 2017; Christison-Lagay and Cohen 2018; Francis et al. 2018b, 2018b;

97 Gronskaya and von der Behrens 2019; Huang et al. 2019; Niwa et al. 2012b). As one progresses further

98 along the auditory cortical hierarchy, there is either an increasingly larger proportion of neurons

99 showing activity correlated to the decision, or the nature of the choice signal changes (Atiani et al. 2014;

100 Niwa et al. 2013; Tsunada et al. 2016). Both cases suggest that the sensory evidence informing task-

101 relevant decisions is transformed as the information moves up the processing stream (Bizley and Cohen

102 2013; Hackett 2011; Huang and Brosch 2020; Romanski et al. 1999).

There has also been uncertainty as to whether the reported choice activity in AC could be more

104 reflective of motor influences than perceptual or decision-related influences. Go/No-Go tasks are

105 typically used in auditory cortical studies, and these tasks require movement for report of one choice, 
but not the other (Brosch 2005; Niwa et al. 2013); forced-choice tasks reduce this uncertainty by requiring movements for either report (Guo et al. 2019). It has been well documented that movement can modulate auditory cortical activity (Eliades and Wang 2003; Guo et al. 2019; Schneider et al. 2014). responses in order to disentangle motor-related from choice-related activity in AC.

112 attention in visual cortex is employed in primary (A1) and secondary (middle lateral belt, ML) auditory

113 cortex using noise that was amplitude modulated (AM) or bandwidth restricted ( $\triangle \mathrm{BW})$. Monkeys were

114 presented sounds that varied either in spectral $(\triangle \mathrm{BW})$ or temporal $(\mathrm{AM})$ dimensions, or both, and

115 performed a detection task in which they reported change along one of these feature dimensions. In this

116 study, we focus on the amplitude modulation feature, as it has been well studied and is a salient

117 communicative sound feature for humans and other animals (Schnupp 2006; Shannon et al. 1995; Van

118 Tasell et al. 1987; Wang et al. 2007) and can be helpful in sound source segregation (Bregman 1994;

119 Grimault et al. 2002). Spectral content changes were used as a difficulty-matched attentional control.

120 We hypothesized we would see a gain in AM encoding when animals were cued to attend to that

121 feature, compared to when they were cued to attend $\Delta \mathrm{BW}$ changes. We also examined choice-related

122 activity in $\mathrm{AC}$, hypothesizing to find a larger proportion of neurons with significant choice-related activity

123 in higher-order $\mathrm{AC}(\mathrm{ML})$ than in $\mathrm{A} 1$.

125 Materials and Methods

126 Subjects.

Subjects were two adult rhesus macaques, one male (13kg, 14-16 years old), one female ( $7 \mathrm{~kg}$, 
129 Use Committee and met the requirements of the United States Public Health Service policy on experimental animal care.

Stimuli.

Stimuli were constructed from broadband Gaussian (white) noise bursts ( $400 \mathrm{~ms} ; 5$ ms cosine ramped), 9 octaves in width ( 40 to $20480 \mathrm{~Hz}$ ). Four different seeds were used to create the carrier noise, which was frozen across trials. To introduce variance along spectral and temporal dimensions, the spectral bandwidth of the noise was narrowed $(\triangle \mathrm{BW})$ and/or the noise envelope was sinusoidally amplitude modulated (AM). The extent of variation in each dimension was manipulated to measure behavioral and neural responses above and below threshold for detecting each feature. Sound generation methods have been previously reported (O'Connor et al., 2011). Briefly,

140 sound signals were produced using an in-house MATLAB program and a digital-to-analog converter

141 (Cambridge Electronic Design [CED] model 1401). Signals were attenuated (TDT Systems PA5, Leader

142 LAT-45), amplified (RadioShack MPA-200), and presented from a single speaker (RadioShack PA-110)

143 positioned approximately $1.5 \mathrm{~m}$ in front of the subject centered at the interaural midpoint. Sounds were 144 generated at a $100 \mathrm{kHz}$ sampling rate. Intensity was calibrated across all sounds (Bruel \& Kjaer model $1452231)$ to $65 \mathrm{~dB}$ at the outer ear. It is important to note that some methods of generating $\Delta \mathrm{BW}$ introduce 146 variation in that sound's envelope, however we implemented a synthesis method that constructs noise 147 using a single-frequency additive technique and thus avoids introducing envelope variations that could 148 serve as cues for $\triangle \mathrm{BW}$ (Strickland and Viemeister 1997).

Recording procedures. 
al. 2005). Placement of the craniotomy was based on stereotactic coordinates of auditory cortex to

154 allow vertical access through parietal cortex to the superior temporal plane (Saleem and Logothetis

155 2007).

All recordings took place in a sound attenuating, foam-lined booth (IAC: $2.9 \times 3.2 \times 2$ meters) while

157 subjects sat in an acoustically transparent chair (Crist Instruments). Three quartz-coated tungsten

158 microelectrodes (Thomas Recording, 1-2 M $2 ; 0.35 \mathrm{~mm}$ horizontal spacing; variable, independently

159 manipulated vertical spacing) were advanced vertically to the superior surface of the temporal lobe.

160 Extracellular signals were amplified (AM Systems model 1800), bandpass filtered between $0.3 \mathrm{~Hz}$ and 10

$161 \mathrm{kHz}$ (Krohn-Hite 3382), and then converted to a digital signal at a $50 \mathrm{kHz}$ sampling rate (CED model

162 1401). During electrode advancement, auditory responsive neurons were isolated by presenting various

163 sounds while the subject sat passively. When at least one auditory responsive single unit was well

164 isolated, we measured neural responses to the two features while the subjects sat passively awake. At

165 least 10 repetitions of each of the following stimuli were presented: the unmodulated noise, each level

166 of bandwidth restriction, and each of the possible AM test modulation frequencies (described below).

167 We also measured pure tone and bandpass noise tuning to aid in distinguishing area boundaries.

168 After completing these tuning measures, experimental behavioral testing and recording began. When

169 possible, tuning responses to the tested stimuli were again measured after task performance, to ensure

170 stability of electrodes throughout the recording. Contributions of single units (SUs) to the signal were

171 determined offline using principal components analysis-based spike sorting tools from Spike2 (CED).

172 Spiking activity was at least $4-5$ times the background noise level. Fewer than $0.1 \%$ of spike events

173 assigned to single unit clusters fell within a $1 \mathrm{~ms}$ refractory period window. Only recordings in which

174 neurons were well isolated for at least 180 trials within each condition were included in analysis here.

Mohn et al. 
Cortical field assessment.

Recording locations were determined using both stereotactic coordinates (Martin and Bowden 1996) and established physiological measures (Merzenich and Brugge 1973; Rauschecker and Tian 2000;

Tian and Rauschecker 2004). In each animal, we mapped characteristic frequency (CF) and sharpness of bandpass noise tuning to establish a topographic distribution of each. Tonotopic gradient reversal, BW distribution, spike latency and response robustness to pure tones was used to estimate the boundary between A1 and ML and assign single units to an area (Downer et al. 2017a; Niwa et al. 2015).

Recordings were assigned to their putative cortical fields post hoc using recording location, tuning preferences, and latencies.

\section{Feature attention task.}

This feature attention task has been previously described in detail (Downer et al. 2017b). The subjects performed a change detection task in which only changes in the attended feature were relevant for the task. Subjects moved a joystick laterally to initiate a trial, wherein an initial sound (the S1, always the 9-octave-wide broadband, unmodulated noise) was presented, followed by a second sound (S2) after a $400 \mathrm{~ms}$ inter-stimulus interval (ISI). The $S 2$ could be identical to the $S 1$, it could change by being amplitude-modulated (AM), it could change by being bandwidth restricted ( $\triangle \mathrm{BW})$, or it could change along both feature dimensions.

Only three values of each feature $(A M, \triangle B W)$ were presented, limiting the size of the stimulus set in order to obtain reasonable power for neural data analysis. The stimulus space was further reduced by presenting only a subset of the possible co-varying stimuli. Within each recording session, we presented 13 total stimuli. To equilibrate difficulty between the two features, we presented values of each feature so that one was near threshold, one was slightly above, and one far above threshold.

Mohn et al. 
Thresholds were determined for each feature independently for each subject using a range of six levels

200 for each feature before three feature values for each animal were selected and the co-varying feature

201 attention task began. For Monkey $\mathrm{U}$, the $\Delta \mathrm{BW}$ values were $0.375,0.5$, and 1 octave and the AM depth

202 values were $28 \%, 40 \%$, and $100 \%$. For Monkey $\mathrm{W}$, the $\Delta \mathrm{BW}$ values were $0.5,0.75$, and 1.5 octaves and

203 the AM depth values were $40 \%, 60 \%$, and $100 \%$.

For all analyses in which data are collapsed across subjects, $\triangle B W$ values and $A M$ values are

presented at only a single modulation frequency. Across sessions, a small set of frequencies was used penalty ( $3-5 \mathrm{~s}$ timeout). 
223 stimulus. Not including instruction trials, block length was at least 180 trials and at most 360 trials, to

224 ensure that subjects performed in each attention condition at least once during the experiment.

225 Subjects could perform each attention condition multiple times within a session. Only sessions in which

226 subjects completed at least 180 trials per condition (excluding instruction trials) were considered for

227 analysis in this study.

228

Neurons' firing rate responses across the range of values were calculated to derive a firing rate

231 function for each feature. Functions were categorized based on whether firing rates increased as the

232 level of the feature increased or decreased ('increasing' vs. 'decreasing' functions). Spike counts (SC)

233 were calculated over the entire 400ms stimulus window. SCs in response to feature-present stimuli were

234 normalized over the entire spike count distribution across both features, including unmodulated noise,

235 for that cell. To characterize this response function, we calculated a feature-selectivity index (FSI) for

236 each feature as follows:

237

$$
F S I_{A M}=\frac{S C_{A M_{>0}, \Delta B W_{0}}-S C_{A M_{0}, \Delta B W_{0}}}{S C_{A M_{>0}, \Delta B W_{0}}+S C_{A M_{0}, \Delta B W_{0}}}
$$

$$
F S I_{B W}=\frac{S C_{\Delta B W_{>0}, A M_{0}}-S C_{\Delta B W_{0}, A M_{0}}}{S C_{\Delta B W_{>0}, A M_{0}}+S C_{\Delta B W_{0}, A M_{0}}}
$$

239 Where $\mathrm{SC}_{x}$ is the mean $\mathrm{SC}$ in response to the given set of stimuli designated by the subscript. A Kruskal-

240 Wallis rank-sum test was preformed between distributions of SCs with the feature-present (feature level

241 greater than 0 ) and those with the feature-absent (feature value of 0 ) to determine the significance of

242 the FSI for each neuron. Cells that had a significant FSI for a given feature were categorized as encoding

243 that feature.

Mohn et al. 


$$
\theta_{i}=2 \pi \frac{t_{i \bmod p}}{p}
$$

Where $t_{i}$ is the time of the spike (in $\mathrm{ms}$ ) relative to the onset of the stimulus and $p$ is the modulation period of the stimulus (in ms). When spike count is low, VS has a tendency to report as spuriously high. Phase projected Vector Strength $\left(\mathrm{VS}_{\mathrm{pp}}\right)$, is a variation on VS developed to help mitigate issues with low SC trials (Yin et al. 2011). VS ${ }_{p p}$ is calculated by first calculating VS for each trial, then the mean phase angle of each trial is compared to the mean phase angle of all trials, and the trial VS value is penalized if out of phase with the global mean response. $V S_{p p}$ is defined as:

$$
V S_{p p}=V S_{t} \cos \left(\phi_{t}-\phi_{c}\right)
$$

Where $V S_{p p}$ is the phase-projected vector strength per trial, $V S_{t}$ is the vector strength per trial, as calculated in [1], and $\phi_{t}$ and $\phi_{c}$ are the trial-by-trial and mean phase angle in radians, respectively, calculated for each stimulus by:

Where $n$ is the number of spikes per trial (for $\phi_{t}$ ) or across all trials (for $\phi_{c}$ ). In this report, we use VS exclusively to measure phase-locking, as SC tended to be relatively low and VS and VS $S_{p p}$ tend to be in good agreement with the exception of low SCs where VS $S_{p p}$ tends to be more accurate than VS (Yin et al. 2011). To determine significance of $\mathrm{VS}_{\mathrm{pp}}$ encoding for each neuron, a Kruskal-Wallis rank-sum test was preformed between distributions of $\mathrm{VS}_{\mathrm{pp}}$ values on trials with non-zero $\mathrm{AM}$ depths, to those from

$$
\phi=\arctan 2\left(\frac{\sum_{i=1}^{n} \sin \theta_{i}}{\sum_{i=1}^{n} \cos \theta_{i}}\right)
$$


this is a control measurement assuming the same modulation frequency as the corresponding AM

269 frequency from that recording session.

\section{Analysis of neural discriminability}

We applied the signal detection theory-based metric area under the receiver operating

273 characteristic (ROCa) (Green and Swets 1974) to measure how well neurons could detect each feature.

274 ROCa represents the probability an ideal observer can detect the presence of the target feature given

275 only a measure of the neural responses (either firing rate or $\mathrm{VS}_{\mathrm{pp}}$ ). To calculate ROCa, we partitioned the 276 trial-by-trial neural responses into two distributions: those when the target feature was present in the

277 stimulus and trials where it was absent. Then we determined the proportion of trials in each group

278 where the neural response exceeds a criterion value. We repeated the measure using 100 criterion

279 values, covering the whole range of responses. The plot of the probability of exceeding the criterion for

280 feature-present trials (neural 'hits') versus the probability of exceeding the criteria for feature-absent

281 trials (neural false alarms) plotted for all 100 criteria as separate points creates the ROC plot. The area

282 under this curve is the ROCa. ROCa is bounded by 0 and 1 , where both extremes indicate perfect

283 discrimination between target feature-present and -absent stimuli, and 0.5 indicates a chance level of

284 discrimination between the two distributions.

Analysis of choice-related activity

288 between neural responses contingent on what the animal reports, for example, whether a stimulus

289 feature is present or absent (Britten et al. 1992, 1996). Similar to ROCa described above, CP values are

290 bounded by 0 and 1 , and a CP value of 0.5 indicates no difference (or perfect overlap) in the neural

291 responses between 'feature-present' and 'feature-absent' reports. A CP value of 1 means for every trial 
292 that the animal reports a feature, the neuron fired more than on trials where the animal did not report

293 the feature. A CP value of 0 means that, for every trial that the animal reports a feature, the neuron

294 fired less than on trials where the animal did not report the feature. Stimuli that did not have at least 5

295 'yes' and 5 'no' responses were excluded from analyses. CP was calculated based on both firing rate and

296 on VS $\mathrm{pp}_{\mathrm{pp}}$. For rate-based CP, we calculated CP both for each stimulus separately, and pooled across

297 stimuli. We calculated this stimulus-pooled CP by first separating the 'yes' and 'no' response trials within

298 stimulus, then converting these rates into z-scores within a stimulus, then combined these z-scored

299 responses across stimuli. This type of z-scoring has been found to be conservative in estimating CP (Kang

300 and Maunsell 2012). CP was calculated during both the $400 \mathrm{~ms}$ stimulus presentation (S2) and during the

301 response window (RW), the time after stimulus offset and prior to the response (typically $\sim 0.2-3 s$ ). The

302 significance of each neuron's CP was determined using a permutation test (Britten et al. 1996). The

303 neural responses were pooled between the 'feature-present report' and 'feature-absent report'

304 distributions and random samples were taken (without replacement). CP was then calculated from this

305 randomly sampled set. This procedure was repeated 2000 times. The $p$ value is the proportion of CP

306 values from these randomly sampled repeats that were greater than the CP value from the non-shuffled 307 distributions.

\section{Results}

We recorded activity from 92 single units in A1 (57 from Monkey W, 35 from Monkey U) from 33

311 recording sessions and 122 single units in ML (49 from Monkey W, 73 from Monkey U) over 39 recording

312 sessions as animals performed a feature-selective attention task. 

though there was no difference in the proportion of $\triangle B W$ encoding neurons between areas $(32.6 \% A 1$, 29.5\% ML; $p=0.18, \chi 2$ test ) (Table 1 ).

in both $\mathrm{A} 1$ and $\mathrm{ML}$ (Table 1). We also found that nearly half of the neurons in both $\mathrm{A} 1$ and $\mathrm{ML}$

325 encoders 'decreasing' in ML. Among significant $\Delta \mathrm{BW}$ encoders, the population was more evenly split

\section{Vector strength encoding}


338 (Niwa et al. 2013), we found VS $S_{p p}$ to be weaker in ML than A1 (Figure 2, $p<0.05$ at all AM depths,

339 Wilcoxon rank-sum Test). In both $\mathrm{A} 1$ and $\mathrm{ML}$, there was no significant difference in phase-locking $\left(\mathrm{VS}_{\mathrm{pp}}\right)$ between the attend $\mathrm{AM}$ and attend $\triangle \mathrm{BW}$ conditions ( $p>0.05$, signed-rank test, Figure 2).

Feature discriminability and context effects

We used the signal detection theory-based area under the receiver operating characteristic on the neural responses (either firing rate or $\mathrm{VS}_{\mathrm{pp}}$ ). Increases in the levels of both features tended to yield increasing ROCa (A1 AM Spearman rho $=0.15$, BW Spearman rho $=.06$; ML AM Spearman rho $=$ condition on either feature at any level of feature modulation for either A1 (Figure 3a,c) or ML (Figure $3 b, d)$.

We found a similar proportion of neurons in A1 (19.5\%) and ML (26.2\%) with significant choicerelated activity during the stimulus window ( $p=0.31, \chi 2$ test). In both areas, the population of neurons 
was larger than during the stimulus window, and the proportions of neurons were again similar between

the two areas (41.3\% A1, 34.4\% ML, $p=0.41, \chi 2$ test).

In $\mathrm{A} 1$, during the attend $\mathrm{AM}$ condition, $\mathrm{CP}$ values were evenly distributed about 0.5 during both

the stimulus presentation ( $\mathrm{S} 2$ median $\mathrm{CP}=0.50, p=0.87$ signed-rank test) and the response window

(RW median $\mathrm{CP}=0.49, p=0.43$ signed-rank test) (Figure $5 \mathrm{a}, \mathrm{c}$ ). In contrast, during the attend $\Delta \mathrm{BW}$

context, the $\mathrm{CP}$ values tended to be lower than 0.5 during both the stimulus ( $\mathrm{S} 2$ median $\mathrm{CP}=0.49, p=$

(Figure $5 \mathrm{~b}, \mathrm{~d}$ ). That is, during the attend $\Delta \mathrm{BW}$ condition, the population of neurons tended to decrease significant difference in the distribution of CP values in ML neurons between the attend $A M$ and attend 
the population shift to $\mathrm{CP}$ values less than 0.5 in the attend $\triangle \mathrm{BW}$ condition compared to the attend $\mathrm{AM}$

386

387

388 condition.

\section{Discussion}

We found a large proportion of cells in $M L$ that decreased firing rate with increasing AM detectability, similar to previous findings in ML (Johnson et al. 2020; Niwa et al. 2013). However, unlike these previous studies where ML had a significantly larger population of cells with decreasing AM depth functions than $A 1$, we found a similar proportion of $A 1$ neurons with decreasing $A M$ depth functions. Further, the majority of neurons in both $\mathrm{A} 1$ and $\mathrm{ML}$ significantly encoding $\mathrm{AM}$ depth had increasing $\mathrm{AM}$ depth functions. This suggests that the encoding of amplitude modulation can be flexible depending upon the behavioral and sensory demands of the task. In essence, with increased perceptual difficulty, stimulus/feature ambiguity, and task difficulty it may be necessary for A1 to develop a more robust and appropriate code in order to solve the task, and for $M L$ to take on more of the sensory processing, and thus the encoding schemes look more similar between these two areas.

We also found a large population of cells in both A1 and ML that were sensitive to changes in bandwidth. This was particularly surprising as the changes in bandwidth were relatively small compared to the 9-octave wide unmodulated noise. It's possible that the $\Delta \mathrm{BW}$ encoding we saw was due to an increasing concentration of power in the middle frequencies of the broadband noise as the level of bandwidth restriction increased. It could also be caused by decreasing power in flanking inhibitory bands. Further studies investigating if and how neurons in $\mathrm{A} 1$ and $\mathrm{ML}$ encode small changes in spectral bandwidth to broad-band sounds under power-matched conditions could be enlightening. Using phase-projected vector strength (VS $\mathrm{pp}_{\mathrm{pp}}$ ) as a measure of temporal coding, neither $\mathrm{ML}$ nor A1 single neurons showed attention-related changes in $\mathrm{VS}_{\mathrm{pp}}$-based sensitivity to AM or VS-based choicerelated activity. This is consistent with previous results from our lab showing smaller effects for $\mathrm{VS}_{\mathrm{pp}}{ }^{-}$ 
based attention and choice than for firing rate (Niwa et al. 2013). A recent study that could help

411 and possibly attention) relay information about temporally modulated sounds in the form of phase-

412 locking, whereas cortical projections to the striatum only convey information about temporally

413 modulated sounds with average firing rate over the stimulus (Ponvert and Jaramillo 2019).

Attending to the target-feature did not significantly improve single neuron amplitude

417 behavioral contexts (Atiani et al. 2014; Bagur et al. 2018; Buran et al. 2014; Francis et al. 2018a; Niwa et

In macaque monkeys, an improvement in both rate-based and temporal AM encoding was observed in

420 A1 and ML neurons when animals performed a single-feature AM detection task compared to when

421 animals passively listened to the same stimuli (Niwa et al. 2013, 2015). We did not see a similar level of

422 encoding improvement, possibly due to the more fine-tuned form of attention needed to perform this

423 task.

One might expect to observe smaller effects from this more selective form of attention than in a compared to another is much smaller than switching between paying attention to a sound and passive

427 sound presentation. Furthermore, arousal, as measured with pupillometry, has recently been shown to

428 correlate with increases in activity, gain and trial-to-trial reliability of A1 neurons (Schwartz et al. 2019),

429 which could account for some of the effects seen in task engagement paradigms. 
433 al. 2014) found in the encoding of these features (i.e. most neurons are sensitive to both $\mathrm{AM}$ and $\Delta \mathrm{BW}$ ).

434 However, it is likely that mixed-selectivity is not the only reason we did not see a gain effect. In a study

435 where rats performed a frequency categorization task with shifting boundaries, Jaramillo and colleagues

436 similarly found that neurons in AC did not improve their discriminability with attentional context

437 (Jaramillo et al. 2014). This similar lack of enhancement seen in a task where only a single feature is

438 modulated, suggests that the mechanism for feature attention in auditory cortex could be enacted via a

439 different mechanism.

In visual cortical studies probing selective feature attention - where the subject must

distinguish between features within a single object, rather than object- or place-oriented, feature-based

442 attention - results have been similarly complex. At the level of the single neuron, there have not been

443 clear, gain-like improvements in the sensitivity to the attended feature (Chen et al. 2012; Mirabella et al.

444 2007; Sasaki and Uka 2009; Uka et al. 2012). Further, the effects of feature-selective attention seem to

445 be dependent upon not just the tuning preferences of a neuron, but also the strength of its tuning (Ruff

446 and Born 2015). These studies, along with our own, suggest that segregation of features within an object

447 may require a different mechanism relative to object-directed, feature-based attention.

In each of the feature-selective attention studies cited above, a common observation is that

single neurons in sensory cortex have mixed selectivity for the features in the task, as opposed to being

450 uniquely responsive to one feature or another. Such mixed selectivity among single neurons may permit

451 sophisticated, flexible computations at the population level (Fusi et al. 2016). It thus seems likely the

452 mechanism for feature-selective attention lies not at the level of the single neuron, but rather requires

453 the integration of activity from a larger population of neurons. A feature-selective study using ERPs

454 found that the neural responses to identical stimuli varied when the subjects attend to different

455 features of the stimulus (Nobre et al. 2006). The single neuron and neural circuit mechanisms

456 underlying this effect remain unclear. One such possible mechanism might be the structure of

Mohn et al. 
correlated variability within the population, which has been shown to be modulated by feature-selective

458 attention (Downer et al. 2017b). Another study, simulating populations by pooling single-neurons across A1 recordings permitted clear segregation of these two features, as well as an enhancement in discrimination of the attended feature (Downer et al. 2020). Further studies investigating featureselective attention at the level of populations of neurons are necessary to better understand the underlying mechanisms.

We did see an interesting difference in the distribution of choice-related activity between the attentional conditions, where the correlation between firing rate and choice shifted direction between conditions. During the attend AM context, $\mathrm{CP}$ was evenly distributed about 0.5 with some neurons showing significant choice activity at either extreme. In contrast, during the attend BW context, $\mathrm{CP}$ values were shifted towards 0 , with very few neurons having significant choice-related activity greater 468 than 0.5 (increasing firing rate for 'feature-present' response). Neurons in auditory cortical areas may 469 also modulate their responses to motor events (Brosch 2005). Some previous reports on choice-related activity have been difficult to interpret, as they employed a Go/No-Go task format in which one

471 perceptual choice required a movement and the other choice did not (Brosch 2005; Niwa et al. 2013).

472 Therefore, the choice-related activity observed was difficult to disentangle from a general preparation

473 to move. The task reported here was a Yes/No forced-choice task, requiring a motor response to each

474 decision (target present versus target absent). The shift in choice-related activity between attention 475 conditions observed in this force choice task, and another recent study (Guo et al. 2019) shows that this 476 choice-related activity cannot simply reflect motor preparation or action. This then strengthens the 477 possible relationship between this activity and the decision or attention process. suggests one or more of the following: (1) the feature-selective attention required in this task is not

480 implemented at the level of an individual neuron in A1 or ML; (2) the feature-selective attention 
bioRxiv preprint doi: https://doi.org/10.1101/2020 03.10.986349; this version posted June 29, 2020. The copyright holder for this preprint (which was not certified by peer review) is the author/funder, who has granted bioRxiv a license to display the preprint in perpetuity. It is made available under aCC-BY-NC-ND 4.0 International license.

481 necessary for this particular task occurs at a later stage in auditory processing; (3) the mixed selectivity

482 of single neurons in $\mathrm{A} 1$ and $\mathrm{ML}$ for these features complicates the interpretability of the effects of

483 attention at the single neuron level, in contrast to feature-based attention neurons studied found in

484 visual cortex (Martinez-Trujillo and Treue 2004; Maunsell 2015; Maunsell and Treue 2006). While we did

485 not see robust differences in encoding between attentional conditions, the difference in attentional

486 choice-related activity reveals that it is not simply reflective of motor preparation, and suggests that

487 activity correlated to reported choice as early as A1 could be informing perceptual and decision

488 processes.

489

490

491

492

493

494

495

496

497

498

499

500

501

502

Mohn et al. 
bioRxiv preprint doi: https://doi.org/10.1101/2020.03.10.986349; this version posted June 29, 2020. The copyright holder for this preprint (which was not certified by peer review) is the author/funder, who has granted bioRxiv a license to display the preprint in perpetuity. It is made available under aCC-BY-NC-ND 4.0 International license.

504

505

\begin{tabular}{|c|c|c|c|c|c|c|c|c|c|}
\hline & $\begin{array}{c}\text { AM Rate } \\
\text { Coder }\end{array}$ & $\begin{array}{c}\text { Decreasing } \\
\text { AM }\end{array}$ & $\begin{array}{l}\text { Increasing } \\
\text { AM } \\
\text { +AM coder }\end{array}$ & $\begin{array}{c}\text { Decreasing } \\
\text { AM } \\
\text { + AM coder }\end{array}$ & $\begin{array}{l}\text { B.W } \\
\text { Coder }\end{array}$ & $\begin{array}{c}\text { Decreasing } \\
\text { BW }\end{array}$ & $\begin{array}{c}\text { Increasing } \\
\text { BW } \\
+ \text { BW Croder }\end{array}$ & $\begin{array}{c}\text { Decrea sing } \\
\text { BW } \\
+ \text { BW Coder }\end{array}$ & vs Coder \\
\hline $\begin{array}{c}\text { A1 } \\
(n=92)\end{array}$ & $47.8 \%$ & $32.6 \%$ & $41.3 \%$ & $6.5 \%$ & $32.6 \%$ & $42.5 \%$ & $18.5 \%$ & $14.1 \%$ & $32.6 \%$ \\
\hline$\underset{(n=122)}{M L}$ & $38.5 \%$ & $27.1 \%$ & $34.4 \%$ & $4.1 \%$ & $29.5 \%$ & $41.8 \%$ & $20.5 \%$ & $9.0 \%$ & $30.33 \%$ \\
\hline
\end{tabular}

506

A.
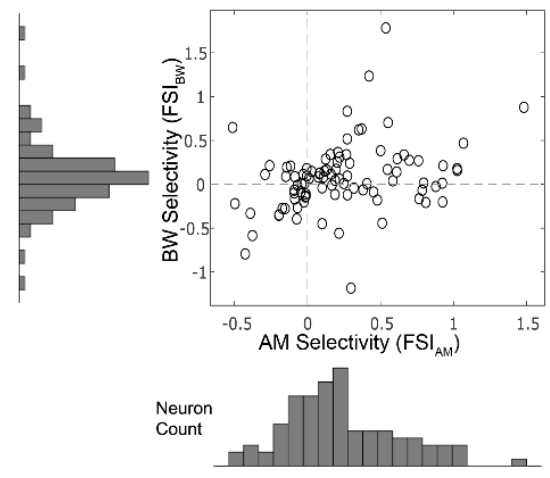

B.

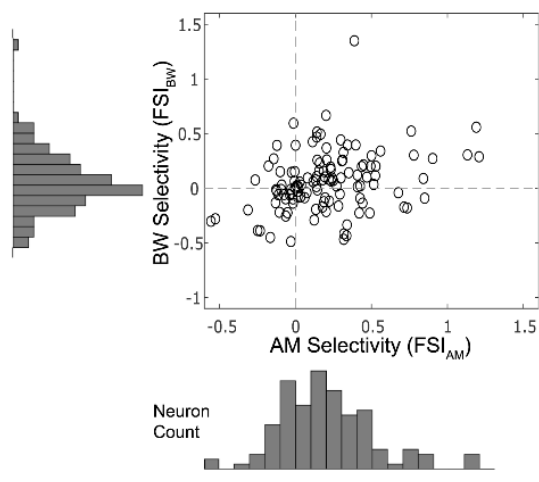

Figure 1: Single neuron feature selectivity index (FSI), a measure of how sensitive a neuron is to changes in each feature value separately. A. A1: a positive correlation between $\mathrm{AM}$ and $\triangle \mathrm{BW}$ selectivity (Pierson rho $=0.3143, p=0.002$ ) B. ML: positive correlation between AM and BW selectivity (Pierson's rho $=0.3109, p=5.32 \mathrm{e}-4$ ) 


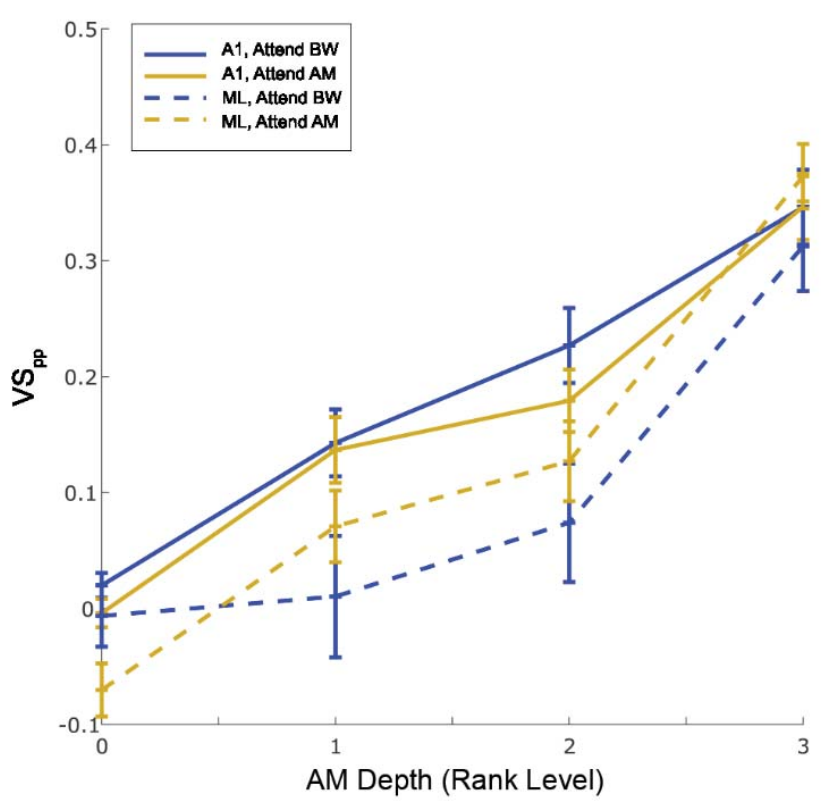

Figure 2. Average phase locking ability of single units in A1 (solid lines) and ML (dashed lines), as measured by phase projected vector strength $\left(\mathrm{VS}_{\mathrm{pp}}\right) . \mathrm{V} \mathrm{S}_{\mathrm{pp}}$ is greater in A1 (solid) than ML (dashed) at low AM depths (AM level 1, $p=0.01 ;$ AM level 2, $p=0.002$, Wilcoxon Ranked Sum), though phase locking is more similar at the highest AM depth ( $p=0.73$, Wilcoxon ranked sum). There was no significant difference in either area between attend $\mathrm{AM}$ (gold) attend $\Delta \mathrm{BW}$ (blue) conditions, $(p>0.05$ for all AM levels, Wilcoxon signed rank test). 

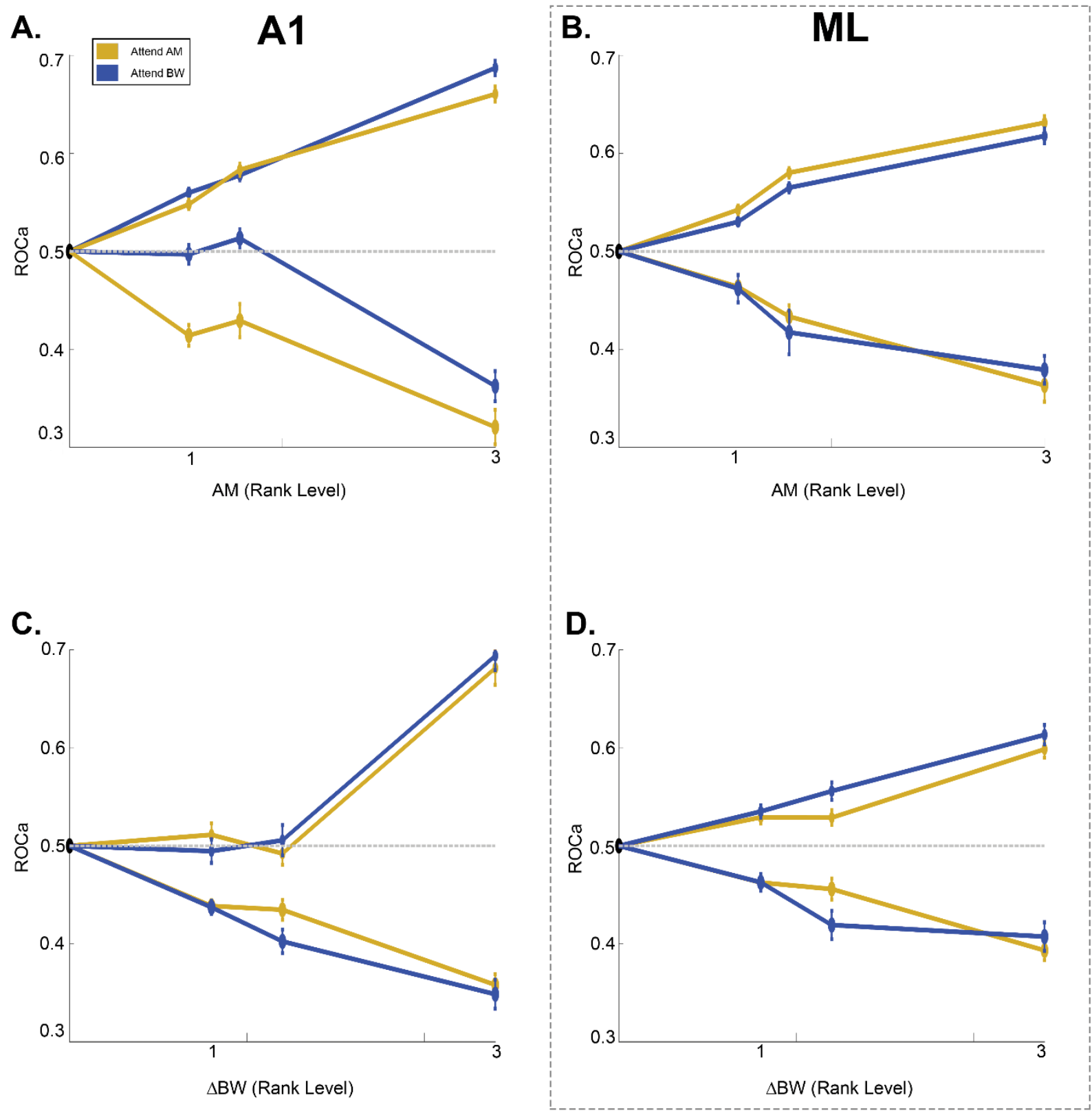

D.

Figure 3: Firing rate based ROCa for each feature by attention condition. Blue lines indicate attend BW condition, yellow lines indicate attend AM condition. A. AM encoding in A1 (38 increasing, 6 decreasing cells). B. AM encoding in ML (42 increasing, 5 decreasing cells) C. BW encoding in A1 (17 increasing, 13 decreasing cells) D. BW encoding in ML (25 increasing, 11 decreasing cells). There was no significant effect of attentional condition on either feature at any level of feature modulation for either $\mathrm{A} 1(\mathbf{A}, \mathbf{C})$ or $\mathrm{ML}(\mathbf{B}, \mathbf{D})$. 

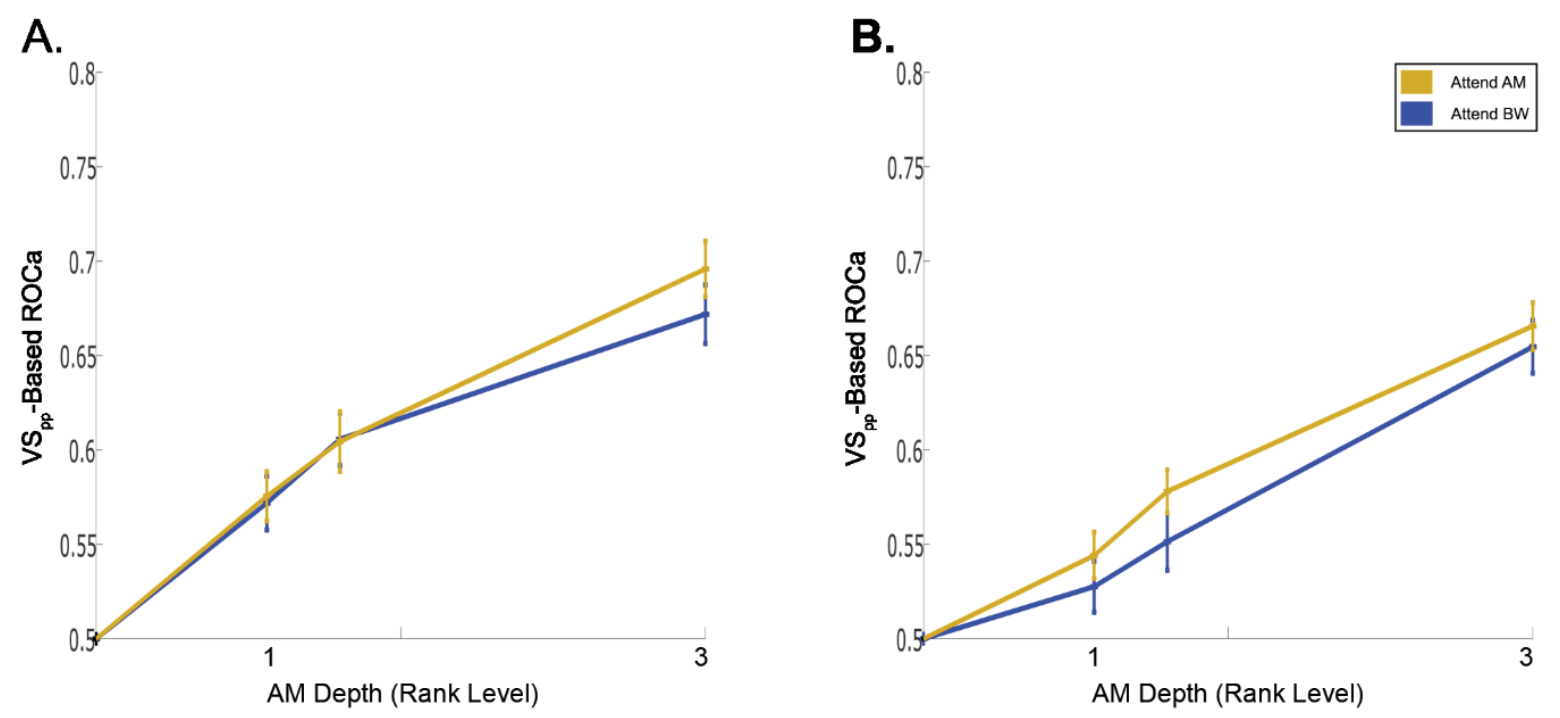

Figure 4: $\mathrm{VS}_{\mathrm{pp}}$-based discriminability (ROCa) of $\mathrm{AM}$ from unmodulated sounds in $\mathrm{A} 1$ and $\mathrm{ML}$ for attend $\mathrm{AM}$ (yellow) and attend $\triangle \mathrm{BW}$ (blue) conditions. A. In $\mathrm{A} 1, \mathrm{VS}_{\mathrm{pp}}$-based discriminability of AM is not significantly different between attention conditions ( $p>0.05$, signed-rank test) B. In $M L, \mathrm{VS}_{\mathrm{pp}}$-based ROCa does not differ between attentional conditions $(p>0.05$, signed-rank test). At low modulation depths (AM depth rank =1). A1 had significantly better AM discriminability than ML ( $p=0.02$, rank sum test), however they were not significantly different at the higher modulation depths (AM ranks 2 and $3, p>0.05$, rank sum test). 
A.

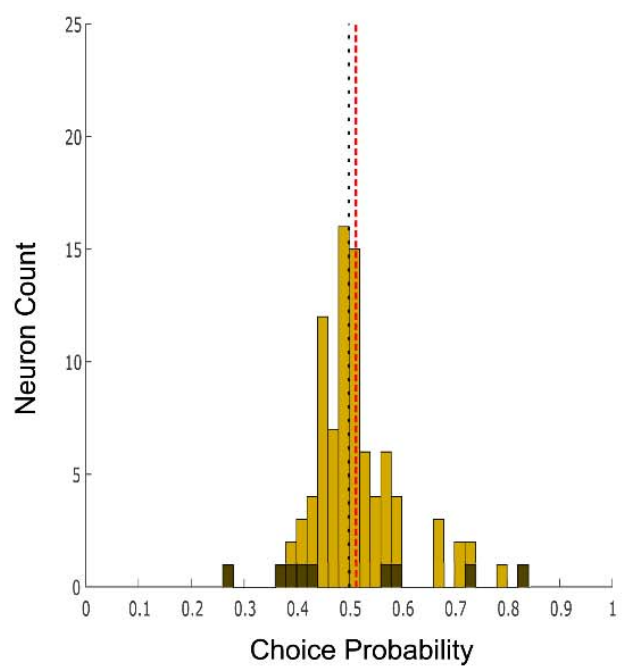

C.

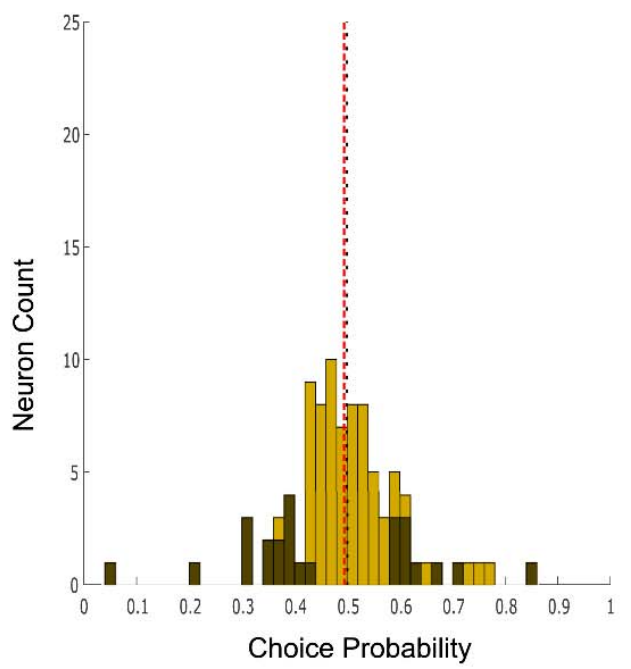

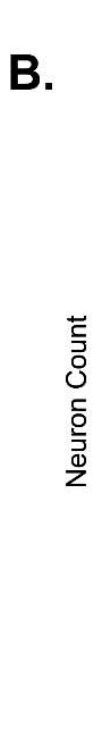
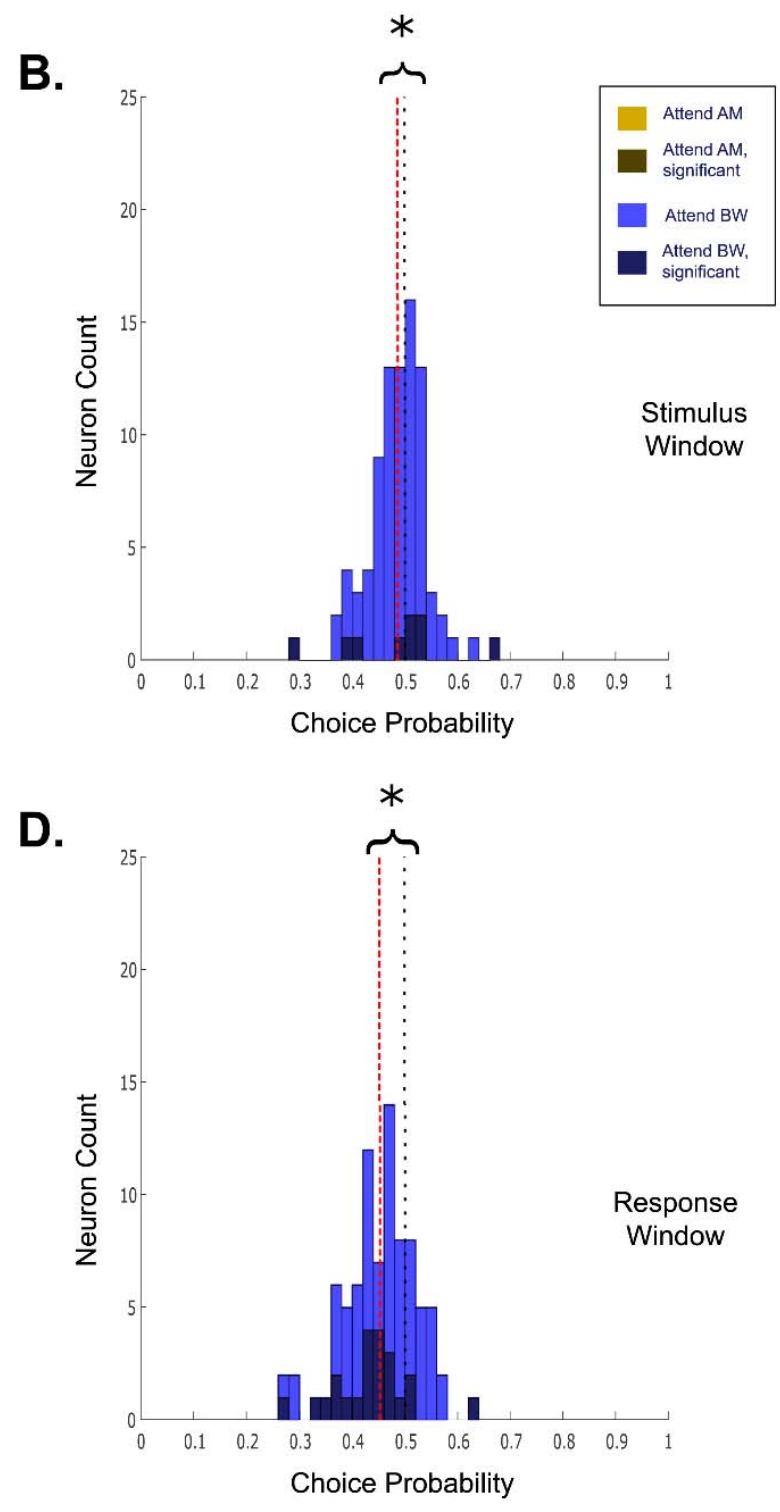

Figure 5: Choice probability in A1. Values closer to 0 indicate increased activity for 'featureabsent' response, whereas 1 indicates increased activity for 'feature-present' response. Darker colored bars indicate cells with significant choice activity. Black dotted line indicates 0.5 , red dashed line denotes the population median. CP during the attend AM condition is evenly distributed about 0.5 in both $A$. the stimulus window (median $=0.50, p=0.87$, signed-rank test) and $C$. the response window (median $=0.49, p=0.43$, signed-rank test). In the attend $\triangle B W$ condition, $C P$ values tended to be less than 0.5 in both $\mathbf{B}$. the stimulus window (median $=0.49, p=0.02$ signed-rank test) and $\mathbf{D}$. the response window (median $=0.46, p=4.2 \mathrm{e}-8$ signed-rank test). There was a significant difference in the population CP distributions between attention conditions during the $\mathrm{RW}(p=0.004$, signedrank test), though not during the $S 2$ ( $p=0.06$, signed-rank test). 

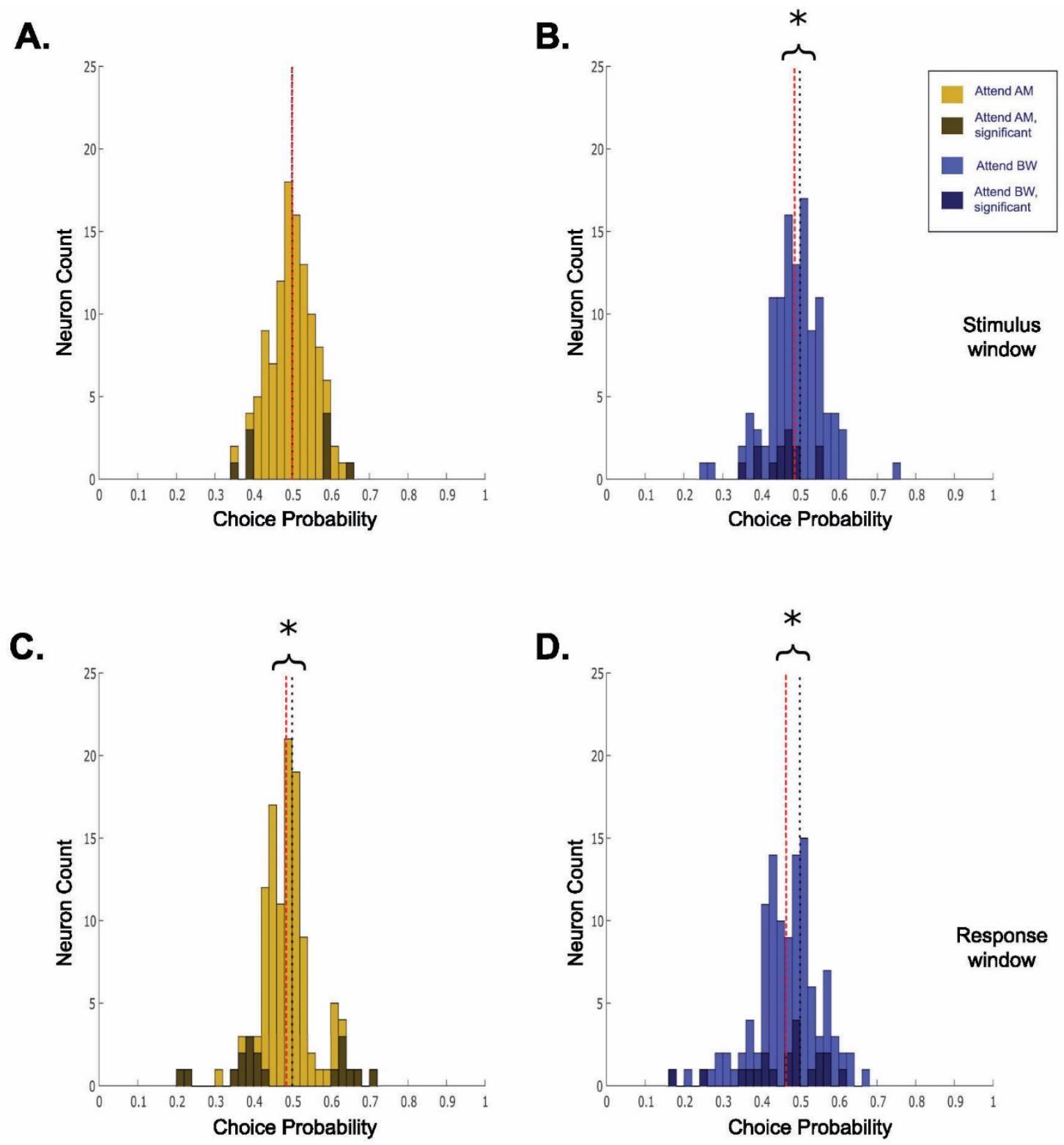

Figure 6: Choice probability in ML, as in Figure 5. CP during the attend AM condition is evenly distributed about 0.5 in $\mathbf{A}$. the stimulus window (median $=0.50, p=0.94$, signed-rank test). However, in the response window C. CP values tended to be less than 0.5 (median $=0.48, p=$ 0.004 , signed-rank test). In the attend $\triangle \mathrm{BW}$ condition, $\mathrm{CP}$ values tended to be less than 0.5 in both $\mathbf{B}$. the stimulus window (median $=0.49, p=0.043$ signed-rank test) and $\mathbf{D}$. the response window (median $=0.47, p=2.7$ e-5 signed-rank test). As in A1, there was a significant difference in the population CP distributions between attention conditions during the RW $(p=$ 0.033 , signed-rank test), though not during the $S 2$ ( $p=0.15$, signed-rank test). 


\section{References}

Angeloni C, Geffen M. Contextual modulation of sound processing in the auditory cortex. Current Opinion in Neurobiology 49: 8-15, 2018.

Atiani S, David SV, Elgueda D, Locastro M, Radtke-Schuller S, Shamma SA, Fritz JB. Emergent Selectivity for Task-Relevant Stimuli in Higher-Order Auditory Cortex. Neuron 82: 486-499, 2014.

Bagur S, Averseng M, Elgueda D, David S, Fritz J, Yin P, Shamma S, Boubenec Y, Ostojic S. Go/No-Go task engagement enhances population representation of target stimuli in primary auditory cortex. Nat Commun 9: 2529, 2018.

Bathellier B, Ushakova L, Rumpel S. Discrete Neocortical Dynamics Predict Behavioral Categorization of Sounds. Neuron 76: 435-449, 2012.

Bizley JK, Cohen YE. The what, where and how of auditory-object perception. Nature Reviews Neuroscience 14: 693-707, 2013.

Bizley JK, Walker KMM, Nodal FR, King AJ, Schnupp JWH. Auditory Cortex Represents Both Pitch Judgments and the Corresponding Acoustic Cues. Current Biology 23: 620-625, 2013.

Bregman AS. Auditory Scene Analysis: The Perceptual Organization of Sound. MIT Press, 1994.

Britten K, Shadlen M, Newsome W, Movshon J. The analysis of visual motion: a comparison of neuronal and psychophysical performance. The Journal of Neuroscience 12: 4745-4765, 1992.

Britten KH, Newsome WT, Shadlen MN, Celebrini S, Movshon JA. A relationship between behavioral choice and the visual responses of neurons in macaque MT. Visual Neuroscience 13: 87-100, 1996.

Brosch M. Nonauditory Events of a Behavioral Procedure Activate Auditory Cortex of Highly Trained Monkeys. Journal of Neuroscience 25: 6797-6806, 2005.

Buran BN, von Trapp G, Sanes DH. Behaviorally Gated Reduction of Spontaneous Discharge Can Improve Detection Thresholds in Auditory Cortex. Journal of Neuroscience 34: 4076-4081, 2014.

Carcea I, Insanally MN, Froemke RC. Dynamics of auditory cortical activity during behavioural engagement and auditory perception. Nat Commun 8: 14412, 2017.

Chambers AR, Hancock KE, Sen K, Polley DB. Online Stimulus Optimization Rapidly Reveals Multidimensional Selectivity in Auditory Cortical Neurons. Journal of Neuroscience 34: 8963-8975, 2014.

Chen X, Hoffmann K-P, Albright TD, Thiele A. Effect of feature-selective attention on neuronal responses in macaque area MT. Journal of Neurophysiology 107: 1530-1543, 2012.

Christison-Lagay KL, Bennur S, Cohen YE. Contribution of spiking activity in the primary auditory cortex to detection in noise. Journal of Neurophysiology 118: 3118-3131, 2017.

Christison-Lagay KL, Cohen YE. The Contribution of Primary Auditory Cortex to Auditory Categorization in Behaving Monkeys. Front Neurosci 12: 601, 2018.

Mohn et al. 
Downer JD, Niwa M, Sutter ML. Task Engagement Selectively Modulates Neural Correlations in Primary Auditory Cortex. Journal of Neuroscience 35: 7565-7574, 2015.

Downer JD, Niwa M, Sutter ML. Hierarchical differences in population coding within auditory cortex. Journal of Neurophysiology 118: 717-731, 2017a.

Downer JD, Rapone B, Verhein J, O'Connor KN, Sutter ML. Feature-Selective Attention Adaptively Shifts Noise Correlations in Primary Auditory Cortex. The Journal of Neuroscience 37: 5378-5392, 2017b.

Downer JD, Verhein JR, Rapone BC, O’Connor KN, Sutter ML. An emergent population code in primary auditory cortex supports selective attention to spectral and temporal sound features. bioRxiv 2020.03.09.984773, 2020.

Elgueda D, Duque D, Radtke-Schuller S, Yin P, David SV, Shamma SA, Fritz JB. State-dependent encoding of sound and behavioral meaning in a tertiary region of the ferret auditory cortex. Nat Neurosci 22: 447-459, 2019.

Eliades SJ, Wang X. Sensory-Motor Interaction in the Primate Auditory Cortex During Self-Initiated Vocalizations. Journal of Neurophysiology 89: 2194-2207, 2003.

Engle JR, Recanzone GH. Characterizing spatial tuning functions of neurons in the auditory cortex of young and aged monkeys: a new perspective on old data. Frontiers in Aging Neuroscience 4: 36, 2013.

Francis NA, Elgueda D, Englitz B, Fritz JB, Shamma SA. Laminar profile of task-related plasticity in ferret primary auditory cortex. Sci Rep 8: 16375, 2018a.

Francis NA, Winkowski DE, Sheikhattar A, Armengol K, Babadi B, Kanold PO. Small Networks Encode Decision-Making in Primary Auditory Cortex. Neuron 97: 885-897.e6, 2018b.

Fritz J, Shamma S, Elhilali M, Klein D. Rapid task-related plasticity of spectrotemporal receptive fields in primary auditory cortex. Nat Neurosci 6: 1216-1223, 2003.

Fritz JB. Differential Dynamic Plasticity of A1 Receptive Fields during Multiple Spectral Tasks. Journal of Neuroscience 25: 7623-7635, 2005.

Fritz JB, Elhilali M, Shamma SA. Adaptive Changes in Cortical Receptive Fields Induced by Attention to Complex Sounds. Journal of Neurophysiology 98: 2337-2346, 2007.

Fusi S, Miller EK, Rigotti M. Why neurons mix: high dimensionality for higher cognition. Current Opinion in Neurobiology 37: 66-74, 2016.

Goldberg JM, Brown PB. Response of binaural neurons of dog superior olivary complex to dichotic tonal stimuli: some physiological mechanisms of sound localization. Journal of Neurophysiology 32: 613-636, 1969.

Green DM, Swets JA. Signal detection theory and psychophysics. Huntington, NY: Krieger Publishing Company, 1974.

Mohn et al. 
Grimault N, Bacon SP, Micheyl C. Auditory stream segregation on the basis of amplitude-modulation rate. The Journal of the Acoustical Society of America 111: 1340-1348, 2002.

Gronskaya E, von der Behrens W. Evoked Response Strength in Primary Auditory Cortex Predicts Performance in a Spectro-Spatial Discrimination Task in Rats. J Neurosci 39: 6108-6121, 2019.

Guo L, Weems JT, Walker WI, Levichev A, Jaramillo S. Choice-Selective Neurons in the Auditory Cortex and in Its Striatal Target Encode Reward Expectation. J Neurosci 39: 3687-3697, 2019.

Hackett TA. Information flow in the auditory cortical network. Hearing Research 271: 133-146, 2011.

Huang Y, Brosch M. Associations between sounds and actions in primate prefrontal cortex. Brain Research 1738: 146775, 2020.

Huang Y, Heil P, Brosch M. Associations between sounds and actions in early auditory cortex of nonhuman primates. elife 8: e43281, 2019.

Jaramillo S, Borges K, Zador AM. Auditory Thalamus and Auditory Cortex Are Equally Modulated by Context during Flexible Categorization of Sounds. Journal of Neuroscience 34: 5291-5301, 2014.

Johnson JS, Niwa M, O'Connor KN, Sutter ML. Amplitude modulation encoding in auditory cortex: Comparisons between the primary and middle lateral belt regions. bio Rxiv , 2020. doi:10.1101/2020.03.05.979575.

Kang I, Maunsell JHR. Potential confounds in estimating trial-to-trial correlations between neuronal response and behavior using choice probabilities. Journal of Neurophysiology 108: 3403-3415, 2012.

Lakatos P, Musacchia G, O’Connel MN, Falchier AY, Javitt DC, Schroeder CE. The Spectrotemporal Filter Mechanism of Auditory Selective Attention. Neuron 77: 750-761, 2013.

Lee C-C, Middlebrooks JC. Auditory cortex spatial sensitivity sharpens during task performance. Nature Neuroscience 14: 108-114, 2011.

Lin P-A, Asinof SK, Edwards NJ, Isaacson JS. Arousal regulates frequency tuning in primary auditory cortex. Proc Natl Acad Sci USA 116: 25304-25310, 2019.

Mardia KV, Jupp PE. Directional statistics. Chichester; New York: J. Wiley, 2000.

Martinez-Trujillo JC, Treue S. Feature-Based Attention Increases the Selectivity of Population Responses in Primate Visual Cortex. Current Biology 14: 744-751, 2004.

Massoudi R, Van Wanrooij MM, Van Wetter SMCI, Versnel H, Van Opstal AJ. Task-related preparatory modulations multiply with acoustic processing in monkey auditory cortex. Eur J Neurosci 39: 1538-1550, 2014.

Maunsell JHR. Neuronal Mechanisms of Visual Attention. Annual Review of Vision Science 1: 373-391, 2015.

Mohn et al. 
Maunsell JHR, Treue S. Feature-based attention in visual cortex. Trends in Neurosciences 29: 317-322, 2006.

McDermott JH. The cocktail party problem. Curr Biol 19: R1024-1027, 2009.

Merzenich MM, Brugge JF. Representation of the cochlear partition on the superior temporal plane of the macaque monkey. Brain Research 50: 275-296, 1973.

Mirabella G, Bertini G, Samengo I, Kilavik BE, Frilli D, Della Libera C, Chelazzi L. Neurons in Area V4 of the Macaque Translate Attended Visual Features into Behaviorally Relevant Categories. Neuron 54: 303318, 2007.

Ni AM, Maunsell JHR. Neuronal Effects of Spatial and Feature Attention Differ Due to Normalization. J Neurosci 39: 5493-5505, 2019.

Niwa M, Johnson JS, O’Connor KN, Sutter ML. Active Engagement Improves Primary Auditory Cortical Neurons' Ability to Discriminate Temporal Modulation. Journal of Neuroscience 32: 9323-9334, 2012 a.

Niwa M, Johnson JS, O’Connor KN, Sutter ML. Activity Related to Perceptual Judgment and Action in Primary Auditory Cortex. Journal of Neuroscience 32: 3193-3210, 2012b.

Niwa M, Johnson JS, O’Connor KN, Sutter ML. Differences between Primary Auditory Cortex and Auditory Belt Related to Encoding and Choice for AM Sounds. Journal of Neuroscience 33: 8378-8395, 2013.

Niwa M, O'Connor KN, Engall E, Johnson JS, Sutter ML. Hierarchical effects of task engagement on amplitude modulation encoding in auditory cortex. Journal of Neurophysiology 113: 307-327, 2015.

Nobre AC, Rao A, Chelazzi L. Selective Attention to Specific Features within Objects: Behavioral and Electrophysiological Evidence. Journal of Cognitive Neuroscience 18: 539-561, 2006.

O’Connor KN, Petkov CI, Sutter ML. Adaptive Stimulus Optimization for Auditory Cortical Neurons. Journal of Neurophysiology 94: 4051-4067, 2005.

Osmanski MS, Wang X. Behavioral Dependence of Auditory Cortical Responses. Brain Topogr 28: 365378, 2015.

Otazu GH, Tai L-H, Yang Y, Zador AM. Engaging in an auditory task suppresses responses in auditory cortex. Nature Neuroscience 12: 646-654, 2009.

Ponvert ND, Jaramillo S. Auditory Thalamostriatal and Corticostriatal Pathways Convey Complementary Information about Sound Features. J Neurosci 39: 271-280, 2019.

Rauschecker JP, Tian B. Mechanisms and streams for processing of "what" and "where" in auditory cortex. Proceedings of the National Academy of Sciences 97: 11800-11806, 2000.

Romanski LM, Tian B, Fritz J, Mishkin M, Goldman-Rakic PS, Rauschecker JP. Dual streams of auditory afferents target multiple domains in the primate prefrontal cortex. Nature Neuroscience 2: 1131-1136, 1999.

Mohn et al. 
647 Ruff DA, Born RT. Feature attention for binocular disparity in primate area MT depends on tuning strength. Journal of Neurophysiology 113: 1545-1555, 2015.

649 Runyan CA, Piasini E, Panzeri S, Harvey CD. Distinct timescales of population coding across cortex. 650 Nature 548: 92-96, 2017.

651 Saleem K, Logothetis Nikos K. A combined MRI and histology atlas of the rhesus monkey brain in 652 sterotaxic coordinates. Burlington, MA: Academic Press, 2007.

Sasaki R, Uka T. Dynamic Readout of Behaviorally Relevant Signals from Area MT during Task Switching. Neuron 62: 147-157, 2009.

655

Schneider DM, Nelson A, Mooney R. A synaptic and circuit basis for corollary discharge in the auditory cortex. Nature 513: 189-194, 2014.

657 Schnupp JWH. Plasticity of Temporal Pattern Codes for Vocalization Stimuli in Primary Auditory Cortex. 658 Journal of Neuroscience 26: 4785-4795, 2006.

659 Schwartz ZP, Buran BN, David SV. Pupil-associated states modulate excitability but not stimulus 660 selectivity in primary auditory cortex. Journal of Neurophysiology 191-208, 2019.

Schwartz ZP, David SV. Focal Suppression of Distractor Sounds by Selective Attention in Auditory Cortex. 662 Cerebral Cortex 28: 323-339, 2018.

663

664

665 666

Scott BH, Malone BJ, Semple MN. Effect of Behavioral Context on Representation of a Spatial Cue in Core Auditory Cortex of Awake Macaques. Journal of Neuroscience 27: 6489-6499, 2007.

Shannon RV, Zeng F-G, Kamath V, Wygonski J, Ekelid M. Speech Recognition with Primarily Temporal Cues. Science 270: 303-304, 1995.

Shinn-Cunningham BG. Object-based auditory and visual attention. Trends in Cognitive Sciences 12: 182-186, 2008.

Strickland EA, Viemeister NF. The effects of frequency region and bandwidth on the temporal modulation transfer function. The Journal of the Acoustical Society of America 102: 1799-1810, 1997.

Sutter ML, Shamma SA. The Relationship of Auditory Cortical Activity to Perception and Behavior. In: The Auditory Cortex, edited by Winer JA, Schreiner CE. Springer US, p. 617-641.

Tian B, Rauschecker JP. Processing of Frequency-Modulated Sounds in the Lateral Auditory Belt Cortex of the Rhesus Monkey. Journal of Neurophysiology 92: 2993-3013, 2004.

von Trapp G, Buran BN, Sen K, Semple MN, Sanes DH. A Decline in Response Variability Improves Neural Signal Detection during Auditory Task Performance. Journal of Neuroscience 36: 11097-11106, 2016.

Treue S, Trujillo JCM. Feature-based attention influences motion processing gain in macaque visual cortex. Nature 399: 575-579, 1999.

Mohn et al. 
680 Tsunada J, Cohen YE. Neural mechanisms of auditory categorization: from across brain areas to within 681 local microcircuits. Frontiers in Neuroscience 8: 161, 2014.

682 Tsunada J, Liu ASK, Gold JI, Cohen YE. Causal contribution of primate auditory cortex to auditory 683 perceptual decision-making. Nature Neuroscience 19: 135-142, 2016.

684 Uka T, Sasaki R, Kumano H. Change in Choice-Related Response Modulation in Area MT during Learning 685 of a Depth-Discrimination Task is Consistent with Task Learning. Journal of Neuroscience 32: 13689$68613700,2012$.

687 Van Tasell DJ, Soli SD, Kirby VM, Widin GP. Speech waveform envelope cues for consonant recognition. 688 The Journal of the Acoustical Society of America 82: 1152-1161, 1987.

689 Wang L, Narayan R, Grana G, Shamir M, Sen K. Cortical Discrimination of Complex Natural Stimuli: Can 690 Single Neurons Match Behavior? Journal of Neuroscience 27: 582-589, 2007.

691 Woods KJP, McDermott JH. Attentive Tracking of Sound Sources. Current Biology 25: 2238-2246, 2015.

692 Yin P, Fritz JB, Shamma SA. Rapid Spectrotemporal Plasticity in Primary Auditory Cortex during Behavior. 693 Journal of Neuroscience 34: 4396-4408, 2014.

694 Yin P, Johnson JS, O'Connor KN, Sutter ML. Coding of Amplitude Modulation in Primary Auditory Cortex. 695 Journal of Neurophysiology 105: 582-600, 2011. 